\title{
DUALITY AND RADON TRANSFORM FOR SYMMETRIC SPACES
}

\author{
BY S. HELGASON ${ }^{1}$ \\ Communicated by G. D. Mostow, July 3, 1963
}

1. The dual space of a symmetric space. Let $S$ be a symmetric space (that is a Riemannian globally symmetric space), and let $I_{0}(S)$ denote the largest connected group of isometries of $S$ in the compact open topology. It will always be assumed that $S$ is of the noncompact type, that is $I_{0}(S)$ is semisimple and has no compact normal subgroup $\neq\{e\}$. Let $l$ denote the rank of $S$; then $S$ contains flat totally geodesic submanifolds of dimension $l$. These will be called planes in $S$.

Let $o$ be any point in $S, K$ the isotropy subgroup of $G=I_{0}(S)$ at $o$ and $\mathfrak{f}_{0}$ and $\mathfrak{g}_{0}$ their respective Lie algebras. Let $\mathfrak{g}_{0}=\mathfrak{l}_{0}+\mathfrak{p}_{0}$ be the corresponding Cartan decomposition of $\mathfrak{g}_{0}$. Let $E$ be any plane in $S$ through $o, a_{0}$ the corresponding maximal abelian subspace of $p_{0}$ and $A$ the subgroup $\exp \left(\mathfrak{a}_{0}\right)$ of $G$. Let $C$ be any Weyl chamber in $\mathfrak{a}_{0}$. Then the dual space of $\mathfrak{a}_{0}$ can be ordered by calling a linear function $\lambda$ on $\mathfrak{a}_{0}$ positive if $\lambda(H)>0$ for all $H \in C$. This ordering gives rise to an Iwasawa decomposition of $G, G=K A N$, where $N$ is a connected nilpotent subgroup of $G$. It can for example be described by

$$
N=\left\{z \in G \mid \lim _{t \rightarrow \infty} \exp (-t H) z \exp (t H)=e\right\},
$$

$H$ being an arbitrary fixed element in $C$. The group $N$ depends on the triple $(o, E, C)$. However, well-known conjugacy theorems show that if $N^{\prime}$ is the group defined by a different triple $\left(o^{\prime}, E^{\prime}, C^{\prime}\right)$ then $N^{\prime}=g N g^{-1}$ for some $g \in G$.

Definition. A horocycle in $S$ is an orbit of a subgroup of the form $g N g^{-1}, g$ being any element in $G$.

Let $t \rightarrow \gamma(t)(t$ real $)$ be any geodesic in $S$ and put $T_{t}=s_{t / 2} s_{0}$ where $s_{\tau}$ denotes the geodesic symmetry of $S$ with respect to the point $\gamma(\tau)$. The elements of the one-parameter subgroup $T_{t}(t$ real $)$ are called transvections along $\gamma$. Two horocycles $\xi_{1}, \xi_{2}$ are called parallel if there exists a geodesic $\gamma$ intersecting $\xi_{1}$ and $\xi_{2}$ under a right angle such that $T \cdot \xi_{1}=\xi_{2}$ for a suitable transvection $T$ along $\gamma$. For each fixed $g \in G$, the orbits of the group $\mathrm{gNg}^{-1}$ form a parallel family of horocycles.

Let $M$ and $M^{\prime}$, respectively, denote the centralizer and normalizer of $A$ in $K$. The group $W=M^{\prime} / M$, which is finite, is called the Weyl group.

1 This work was supported in part by the National Science Foundation, NSF GP-149. 
Proposition 1.1. The group $G$ acts transitively on the set of horocycles in $S$. The subgroup of $G$ which maps the horocycle $N \cdot 0$ into itself equals $M N$.

Let $\hat{S}$ denote the set of horocycles in $S$. Then we have the natural identifications

$$
S=G / K, \quad \hat{S}=G / M N
$$

the latter of which turns $\hat{S}$ into a manifold, which we call the dual space of $S$.

Proposition 1.2.

(i) The mapping

$$
\phi:(k M, a) \rightarrow k a K
$$

is a differentiable mapping of $(K / M) \times A$ onto $S$ and a regular w-toone mapping of $(K / M) \times A^{\prime}$ onto $S^{\prime}$.

(ii) The mapping

$$
\hat{\phi}:(k M, a) \rightarrow k a M N
$$

is a diffeomorphism of $(K / M) \times A$ onto $\hat{S}$.

In statement (i) which is well known, $w$ denotes the order of $W$, $A^{\prime}$ is the set of regular elements in $A$ and $S^{\prime}$ is the set of points in $S$ which lie on only one plane through $o$.

Proposition 1.3. The following relations are natural identifications of the double coset spaces on the left:

(i) $K \backslash G / K=A / W$;

(ii) $M N \backslash G / M N=A \times W$.

Statement (i) is again well known; (ii) is a sharpening of the lemma of Bruhat (see [6]) which identifies $M A N \backslash G / M A N$ with $W$.

The proofs of these results use the following lemma.

\section{LEMMA 1.4.}

(i) Let $s_{0}$ denote the geodesic symmetry of $S$ with respect to $o$ and let $\theta$ denote the involution $g \rightarrow s_{0} g s_{0}$ of $G$. Then

$$
(N \theta(N)) \cap K=\{e\} .
$$

(ii) Let $C$ and $C^{\prime}$ be two Weyl chambers in $\mathfrak{a}_{0}$ and $G=K A N$, $G=K A N^{\prime}$ the corresponding Iwasawa decompositions. Then

$$
\left(N N^{\prime}\right) \cap(M A)=\{e\} .
$$

2. Invariant differential operators on the space of horocycles. For any manifold $V, C^{\infty}(V)$ and $C_{c}^{\infty}(V)$ shall denote the spaces of $C^{\infty}$ 
functions on $V$ (respectively, $C^{\infty}$ functions on $V$ with compact support). Let $\boldsymbol{D}(S)$ and $\boldsymbol{D}(\hat{S})$, respectively, denote the algebras of all $G$ invariant differential operators on $S$ and $\hat{S}$. Let $S\left(\mathfrak{a}_{0}\right)$ denote the symmetric algebra over $\mathfrak{a}_{0}$ and $J\left(\mathfrak{a}_{0}\right)$ the set of $W$-invariants in $S\left(\mathfrak{a}_{0}\right)$. There exists an isomorphism $\Gamma$ of $\boldsymbol{D}(S)$ onto $J\left(\mathfrak{a}_{0}\right)$ (cf. [7, Theorem 1, p. 260], also [9, p. 432]). To describe $D(\hat{S})$, consider $\hat{S}$ as a fibre bundle with base $K / M$, the projection $p: \hat{S} \rightarrow K / M$ being the mapping which to each horocycle associates the parallel horocycle through 0 . Since each fibre $F$ can be identified with $A$, each $U \in S\left(\mathfrak{a}_{0}\right)$ determines a differential operator $U_{F}$ on $F$. Denoting by $f \mid F$ the restriction of a function $f$ on $\hat{S}$ to $F$ we define an endomorphism $D_{U}$ on $C^{\infty}(\hat{S})$ by

$$
\left(D_{U} f\right) \mid F=U_{F}(f \mid F) \quad f \in C^{\infty}(\hat{S}),
$$

$F$ being any fibre. It is easy to prove that the mapping $U \rightarrow D_{U}$ is a homomorphism of $S\left(\mathfrak{a}_{0}\right)$ into $D(\hat{S})$.

Theorem 2.1. The mapping $U \rightarrow D_{U}$ is an isomorphism of $S\left(\mathfrak{a}_{0}\right)$ onto $\boldsymbol{D}(\hat{S})$. In particular, $\boldsymbol{D}(\hat{S})$ is commutative.

Although $G / M N$ is not in general reductive, $D(\hat{S})$ can be determined from the polynomial invariants for the action of $M N$ on the tangent space to $G / M N$ at $M N$ (cf. [8, Theorem 10]). It is then found that the algebra of these invariants is in a natural way isomorphic to $S\left(\mathfrak{a}_{0}\right)$, whereupon Theorem 2.1 follows. Let $\hat{\Gamma}$ denote the inverse of the mapping $U \rightarrow D_{U}$.

3. The Radon transform. Let $\xi$ be any horocycle in $S, d s_{\xi}$ the volume element on $\xi$. For $f \in C_{c}^{\infty}(S)$ put

$$
\hat{f}(\xi)=\int_{\xi} f(s) d s_{\xi}, \quad \xi \in \hat{S} .
$$

The function $\hat{f}$ will be called the Radon transform of $f$.

THEOREM 3.1. The mapping $f \rightarrow \hat{f}$ is a one-to-one linear mapping of $C_{c}^{\infty}(S)$ into $C_{c}^{\infty}(\hat{S})$.

Now extend $\mathfrak{a}_{0}$ to a Cartan subalgebra $\mathfrak{h}_{0}$ of $\mathfrak{g}_{0}$; of the corresponding roots let $P_{+}$denote the set of those whose restriction to $\mathfrak{a}_{0}$ is positive (in the ordering defined by $C$ ). Put $\rho=\frac{1}{2} \sum_{\alpha \in P_{+}} \alpha$ and let $p \rightarrow$ ' $p$ denote the unique automorphism of $S\left(\mathfrak{a}_{0}\right)$ given by ' $H=H-\rho(H)$ $\left(H \in \mathfrak{a}_{0}\right)$ (cf. [7, p. 260]).

Theorem 3.2. Let ' $D(\hat{S})$ be given by

$$
{ }^{\prime} D(\hat{S})=\left\{E \in D(\hat{S}) \mid '(\hat{\Gamma}(E)) \in J\left(\mathfrak{a}_{0}\right)\right\},
$$


and let $D \rightarrow \hat{D}$ denote the isomorphism of $D(S)$ onto $D(\hat{S})$ such that

$$
'(\hat{\Gamma}(\hat{D}))=\Gamma(D), \quad D \in D(S) .
$$

Then

$$
(D f)^{\wedge}=\hat{D} \hat{f} \text { for } f \in C_{c}^{\infty}(S) .
$$

In view of the duality between points and horocycles there is a natural dual to the transform $f \rightarrow \hat{f}$. This dual transform associates to each function $\psi \in C^{\infty}(\hat{S})$ a function $\check{\psi} \in C^{\infty}(S)$ given by

$$
\check{\psi}(p)=\int_{\xi \cap p=p} \psi(\xi) d m(\xi), \quad p \in S,
$$

where the integral on the right is the average of $\psi$ over the (compact) set of horocycles passing through $p$. We put

$$
I_{f}=(\hat{f})^{2}, \quad f \in C_{c}^{\infty}(S)
$$

and wish to relate $f$ and $I_{f}$.

TheOREM 3.3. Suppose the group $G=I_{0}(S)$ is a complex Lie group. Then

$$
\square I_{f}=c f, \quad f \in C_{c}^{\infty}(S),
$$

where $c$ is a constant $\neq 0$ and $\square$ is a certain operator in $D(S)$, both independent of $f$.

We shall now indicate the definition of $\square$. Let $J$ denote the complex structure of the Lie algebra $\mathfrak{g}_{0}$. Then the Cartan subalgebra $\mathfrak{h}_{0}$ above can be taken as $\mathfrak{a}_{0}+J \mathfrak{a}_{0}$ and can then be considered as a complex Cartan subalgebra of $\mathfrak{g}_{0}$ (considered as a complex Lie algebra). Let $\Delta^{\prime}$ denote the corresponding set of nonzero roots and for each $\alpha \in \Delta^{\prime}$ select $H_{\alpha}^{\prime}$ in $\mathfrak{h}_{0}$ such that $B^{\prime}\left(H_{\alpha}^{\prime}, H\right)=\alpha(H)\left(H \in \mathfrak{h}_{0}\right)$ where $B^{\prime}$ denotes the Killing form of the complex algebra go. Then $H_{\alpha}^{\prime} \in \mathfrak{a}_{0}$ and the element $\prod_{\alpha \in \Delta^{\prime}} H_{\alpha}^{\prime}$ in $S\left(\mathfrak{a}_{0}\right)$ is invariant under the Weyl group $W$. Then $\square$ is the unique element in $D(S)$ such that

$$
\Gamma(\square)=\prod_{\alpha \in \Delta^{\prime}} H_{\alpha}^{\prime} .
$$

The proof of Theorem 3.3 is based on Theorem 3 in Harish-Chandra [5] (see also Gelfand-Naimark [4, p. 156]), together with the Darboux equation for $S$ ([9, p. 442]). In the case when $S$ is the space of positive definite Hermitian $n \times n$ matrices a formula closely related 
to (1) was given in Gelfand [1]. Radon's classical problem of representing a function in $R^{n}$ by means of its integrals over hyperplanes was solved by Radon [13] and John [10]. Generalizations to Riemannian manifolds of constant curvature were given by Helgason [8], Semyanistyi [15] and Gelfand-Graev-Vilenkin [3].

4. Applications to invariant differential equations. We shall now indicate how Theorem 3.3 can be used to reduce any $G$-invariant differential equation on $S$ to a differential equation with constant coefficients on a Euclidean space. The procedure is reminiscent of the method of plane waves for solving homogeneous hyperbolic equations with constant coefficients (see John [11]).

Definition. A function on $S$ is called a plane wave if there exists a parallel family $\Xi$ of horocycles in $S$ such that (i) $S=\cup_{\xi \in \Xi} \xi$; (ii) For each $\xi \in \Xi, f$ is constant on $\xi$.

Theorem 3.3 can be interpreted as a decomposition of an arbitrary function $f \in C_{c}^{\infty}(S)$ into plane waves.

Now select $g \in G$ such that $\Xi$ is the family of orbits of the group $\mathrm{gNg}^{-1}$. The manifold $\mathrm{gAg}^{-1} \cdot o$ intersects each horocycle $\xi \in \Xi$ orthogonally. A plane wave $f$ (corresponding to $\Xi$ ) can be regarded as a function $f^{*}$ on the Euclidean space $A$. If $D \in D(S)$, then $D f$ is also a plane wave (corresponding to $\Xi$ ) and $(D f)^{*}=D_{A} f^{*}$, where $D_{A}$ is a differential operator on $A$. Using the fact that $a N a^{-1} \subset N$ for each $a \in A$ it is easily proved (cf. [7, Lemma 3, p. 247] or [12, Theorem 1]) that $D_{A}$ is invariant under all translations on $A$. Thus an invariant differential equation in the space of plane waves (for a fixed $\Xi$ ) amounts to a differential equation with constant coefficients on the Euclidean space $A$. Using Theorem 3.3, and the fact that $\square$ commutes elementwise with $D(S)$, an invariant differential equation for arbitrary functions on $S$ can be reduced to a differential equation with constant coefficients (and is thus, in principle, solvable).

EXAMPLE: THE WAVE EQUATION on $S$. For an illustration of the procedure above we give now an explicit global solution of the wave equation on $S\left(I_{0}(S)\right.$ assumed complex).

Let $\Delta$ denote the Laplacian on $S$ and let $f \in C_{c}^{\infty}(S)$. Consider the differential equation

$$
\Delta u=\frac{\partial^{2} u}{\partial t^{2}}
$$

with initial data

$$
u(p, 0)=0 ; \quad\left\{\frac{\partial}{\partial t} u(p, t)\right\}_{t=0}=f(p) \quad(p \in S) .
$$


Let $\Delta_{\boldsymbol{A}}$ denote the Laplacian on $A$ (in the metric induced by $E$ ), $\|\rho\|$ the length of the vector $\rho$ in $\S 3$. Given $a \in A$, let $\log a$ denote the unique element $H \in \mathfrak{a}_{0}$ for which $\exp H=a$. For simplicity, let $e^{\rho}$ denote the function $a \rightarrow e^{\rho(\log a)}$ on $A$. Let $\xi$ denote the horocycle $N \cdot o$.

Given $x \in G, k \in K$, consider the function

$$
F_{k, x}(a)=\int_{\xi} f(x k a \cdot s) d s_{\xi} \quad(a \in A)
$$

and the differential equation on $A \times R$,

$$
\left(\Delta_{A}-\|\rho\|^{2}\right) V_{k, x}^{t}=\frac{\partial^{2}}{\partial t^{2}} V_{k, x}^{t},
$$

with initial data

$$
V_{k, x}^{0}=0 ; \quad\left\{\frac{\partial}{\partial t} V_{k, x}^{t}\right\}_{t=0}=e^{\rho} F_{k, x} .
$$

Equation (3) is just the equation for damped waves in the Euclidean space $A$ and is explicitly solvable (see e.g. [14, p. 88]). The solution of (1) is now given by

$$
u(p, t)=c \quad \square_{p}(V(p, t)),
$$

where

$$
V(x K, t)=\int_{K} V_{k, x}^{t}(e) d k .
$$

Here $d k$ is the normalized Haar measure on $K$ and $c$ is the same constant as in Theorem 3.3. It is not hard to see that the integral in (4) is invariant under each substitution $x \rightarrow x u(u \in K)$ so the function $V(p, t)$ is indeed well defined.

\section{REFERENCES}

1. I. M. Gelfand, Integral geometry and its relation to the theory of group representations, Uspehi Mat. Nauk 15 (1960), 155-164 = Russian Math. Surveys, 1960.

2. I. M. Gelfand and M. I. Graev, The geometry of homogeneous spaces, group representations in homogeneous spaces and questions in integral geometry related to them. I, Trudy Moskov. Mat. Obšc. 8 (1959), 321-390. (Russian)

3. I. M. Gelfand, M. I. Graev and N. Vilenkin, Integral geometry and its relation to problems in the theory of group representations, Vol. 5, Generalized Functions, Moscow, 1962.

4. I. M. Gelfand and M. A. NaYmark, Unitary representations of the classical groups, Trudy Mat. Inst. Steklov. 36(1950); German transl., Akademie Verlag, Berlin, 1957. 
5. Harish-Chandra, The Plancherel formula for complex semisimple Lie groups, Trans. Amer. Math. Soc. 76 (1954), 485-528.

6. - On a lemma of Bruhat, J. Math. Pures Appl. 35 (1956), 203-210.

7. - Spherical functions on a semisimple Lie group. I, II. Amer. J. Math. 80 (1958), 241-310, 553-613.

8. S. Helgason, Differential operators on homogeneous spaces, Acta Math. 102 (1959), 239-299.

9. - Differential geometry and symmetric spaces, Academic Press, New York, 1962.

10. F. John, Bestimmung einer Funktion aus ihren Integralen über gewisse Mannigfaltigkeiten, Math. Ann. 100 (1934), 488-520.

11. - Plane waves and spherical means, applied to partial differential equations, Interscience, New York, 1955.

12. F. I. Karpelevič, Orispherical radial parts of Laplace operators on symmetric spaces, Dokl. Akad. Nauk SSSR 143 (1962), 1034-1037 = Soviet Math. Dokl. 3 (1962), 528-531.

13. J. Radon, Über die Bestimmung von Funktionen durch ihre Integralwerte längs gewisser Mannigfaltigkeiten, Ber. Verh. Sächs. Akad. Wiss. Leipzig Math.-Nat. Kl. 69 (1917), 262-277.

14. M. Riesz, L'integrale de Riemann-Liouville et le problème des Cauchy, Acta Math. 81 (1948), 1-223.

15. V. I. Semyanistyi, Homogeneous functions and some problems of integral geometry in spaces of constant curvature, Dokl. Akad. Nauk SSSR 136 (1961), 288-291 = Soviet Math. Dokl. 2 (1961), 59-62.

Massachusetts Institute of Technology 